DOI: 10.17805/ggz.2019.1.7

\title{
Нормативная и дескриптивная неопределенность в геномной медицине
}

\author{
С. Ю. Шевченко \\ Институт философии РАН, \\ А. Г. Шестак
}

Российский научный центр хирургии имени академика Б. В. Петровского

В статье рассматриваются два вида неопределенности (дескриптивная и нормативная) и эффекты их взаимоналожения в геномной медииине. Дескриптивная неопределенность может быть устранена за счет получения новой информации о некотором положении дел, нормативная же не может быть редуцирована таким образом, она связана с тем, что действующий агент не может осуществить выбор среди противоречащих иенностных оснований своего действия. В рамках геномной медицины источниками дескриптивной неопределенности являются: a) устранимое неведение, незнание каких-либо аспектов медицинкой науки; b) неведение, вызванное отсутствием достаточно точных способов узнать или распознать положение дел (например, наличием критически важных погрешностей средств диагностики); с) стохастический характер прочесса, о будущем течении которого необходимо вынести суждение. Нормативная неопределенность часто возникает в рамках коммуникачии, связанной с дескриптивной неопределенностью. Конфликтующими изенностными основаниями могут выступать «этика выбора» и «этика заботыл», отталкиваясь от которых врач может сообщить или не сообщить пациенту информаџию о неясных степенях медицинского риска.

Нормативная неопределенность не может быть минимизирована за счет получения новой фактической информаџии, дескриптивная неопределенность также не может быть совсем устранена из медицинских практик. Врач и пациент имеют возможность совместно «справиться», «совладать» с обоими видами неопределенности через обращение к этике неопределенности. Основными ее ценностными ориентирами служат устойчивость, благополучие, автономия и солидарность.

Ключевые слова: этика неопределенности; медико-генетическое консультирование; коммуникачия; автономия пациента; смысл долженствования

${ }^{*}$ Статья подготовлена при финансовой поддержке РНФ в рамках проекта № 18-78-10132. The article was prepared with financial support from the Russian Science Foundation (project No. 18-78-10132). 


\title{
Normative and Descriptive Uncertainty in Genomic Medicine
}

\author{
S. Yu. Shevchenko \\ Institute of Philosophy, Russian Academy of Sciences, \\ A. G. Shestak \\ Petrovsky National Research Centre of Surgery
}

We discuss two types of uncertainty (descriptive and normative) and the effects of their overlap in genomic medicine. Descriptive uncertainty can be reduced by obtaining new information about a certain state of affairs. Normative uncertainty cannot be reduced so as it appears due to the fact that the current agent cannot choose among conflicting ethical grounds of action. In the framework of genomic medicine, the following sources of descriptive uncertainty can be mentioned: a) avoidable ignorance, ignorance of any aspects of medical science; b) ignorance caused by the lack of sufficiently accurate ways to know or recognize the state of affairs (for example, the presence of critical diagnostic errors); c) stochastic nature of the predicted process. Normative uncertainty often arises in the context of communication related to descriptive uncertainty. The conflicting ethical grounds of action are 'ethics of choice' and 'ethics of care'. Relying on these principles a doctor can inform or not inform the patient about unclear degrees of medical risk.

While normative uncertainty cannot be minimized by obtaining new factual information, descriptive uncertainty also cannot be completely eliminated from medical practices. A doctor and patient have an opportunity to jointly 'cope' with both types of uncertainty through an appeal to the ethics of uncertainty. Its main values are resilience, welfare, autonomy and solidarity.

Keywords: ethics of uncertainty; genetic counseling; communication; patient autonomy; meaning of 'ought'

\section{ВВЕДЕНИЕ}

Врач и пациент постоянно имеют дело с неопределенностью. Обоими, как правило, она воспринимается как нечто нежелательное. В глазах обоих она может быть связана с чем-то неприемлемым: недостаточными знаниями врача, плохо проведенной диагностикой, неисполнением со стороны пациента данных ему рекомендаций. Следующий шаг в анализе источников неопределенности уже не предполагает быстрых моральных оценок: он связан с признанием нередуцируемой сложности человеческого организма вкупе с невычислимым разнообразием его реакций на факторы внешней среды. Но и в этом случае неопределенность зачастую затушевывается и в научных работах, и во время врачебного приема. Однако в последние несколько лет тема неопределенности в медицине поднимается все чаще в профессиональных изданиях для врачей - особенно часто в связи с развитием современных молекулярно-биологических предиктивных технологий, большая часть из которых относится к сфере геномной медицины.

В настоящей статье мы в сжатой форме рассмотрим философский аспект соотношения дескриптивной и нормативной неопределенностей, про- 
следим, каким образом они актуализируются в медицинской практике и какие средства для их преодоления предлагаются с регуляторной и методологической позиций. Затем мы обозначим, как эти формы неопределенности (обычно рассматриваемые в разных дискурсах) пересекаются в рамках геномной медицины, и рассмотрим этические ориентиры взаимодействия врача и пациента, заданные условиями такой неопределенности.

\section{ДЕСКРИПТИВНАЯ И НОРМАТИВНАЯ НЕОПРЕДЕЛЕННОСТЬ В (МЕТА)ЭТИКЕ}

В аналитической философии проблема неопределенности часто рассматривается в наиболее подходящей для нашего последующего обсуждения форме. А именно, как проблема долженствования в условиях неопределенности. С начала 1990-х гг. ее обсуждение строится вокруг мысленного эксперимента, предложенного специалистом по этике Фрэнком Джексоном (Jackson, 1991). Приведем пересказ версии этого мысленного эксперимента, изложенной в более поздней статье Питтарда и Ворснипа (Pittard, Worsnip, 2017: 156157):

Гуляя по лесу, Ханна, врач, видит человека, укушенного змеей. С собой у нее есть три препарата А, В и С, среди которых она должна выбрать, какой применить, чтобы спасти жизнь укушенному. Ханна не является специалистом в токсикологии, но она знает, что $C$ поможет пострадавшему $и$ при этом вызовет стойкую, но умеренную гиперчувствительность к солнечнылм лучам. А или В могут спасти укушенного без побочных эффектов, но Ханна не помнит, какой именно препарат окажет такое действие. $B$ то же время она знает, что один из этих препаратов (А или В) способен убить пострадавшего. Боясь такого исхода, Ханна заключает: "Я долэна применить С». И делает это. Однако на следуюший день профессор, обучавший Ханну, усльшив эту историю заключает: «Ты должна была применить В. Он бы спас пострадавшего без побочных эффектов. Теперь этот человек не сможет выйти на улииу без защитного крема».

Дискуссии вокруг этого сюжета, как правило, связаны с вопросом, имеет ли слово «должна» (в оригинале 'ought') в устах Ханны и в устах профессора разные значения. И если нет - почему у нас есть два разных верных выражения долженствования для одного и того же действия в одних и тех же обстоятельствах? Разработанная Ангелиной Кратцер семантика позволяет рассмотреть два измерения значения долженствования. Ради краткости изложения, несколько упрощая техническую часть, опишем их скорее для демонстрации усилий в сфере формальной философии, направленных на разрешение проблемы долженствования. Два измерения этой семантики Кратцер предложила называть модальным основанием и источником долженствования. Первое определяет класс возможных миров, в котором долженствование исполнено. Второй выбирает среди этого класса те миры, в которых условия выполнения долженствования наиболее точно соответствуют подразумеваемым в нем условиям. К числу таких условий, несомненно принадлежит знание, которым располагал субъект в момент принятия решения об оптималь- 
ном действии (Dowell, 2012). Именно этим Ханна и отличается от профессора, хотя они и согласны относительно «стандартов» оценки действия в представленном случае.

Иными словами, получение новой информации способно изменить выбор должного действия в конкретной ситуации, при том, что само выражение долженствования, от исполнения которого отказались, остается верным. К примеру, врач знает, что при инфекционном заболевании Z лучшим средством является антибиотик А. Но он узнает также, что у конкретного пациента аллергия на это лекарство, и он прописывает ему препарат В. При этом принцип «A - лучшее средство при Z» не отвергается этим врачом.

Специалист в области метаэтики из Йельского университета Джон Питтард, рассматривая проблему долженствования, предлагает различать нормативную и дескриптивную неопределенность. В рассмотренных выше примерах с укусом змеи и болезнью Z речь идет о последней. Новая информация о положении дел способна поменять решение в условиях дескриптивной неопределенности без отказа от совокупности руководящих принципов. При нормативной неопределенности агент не может совершить выбор между разными руководящими принципами. Питтард и его коллега Алекс Ворснип приводят следующий пример нормативной неопределенности (передадим его с некоторыми сокращениями, опуская специальную терминологию):

Грэг, политик, управленец должен выбрать между тремя налоговыми режимами. Режим А основывается на утилитаристских представлениях об оптимальности и предполагает введение минимальных налоговых ставок в расчете на то, что вся экономика в результате будет работать предельно эффрективно. Режим В основывается на принципах справедливости (близких к сформулированным Джсоном Ролзом), предполагающих максимальное перераспределение ресурсов для помощуи наиболее бедным, и соответственно предусматривает введение более высоких налогов. Согласно этому режиму перераспределения проблемы менее бедных могут быть решены только после того, как улучшилось положение наиболее обездоленных, и т. д. - вверх по лестнице достатка. Режим С представляет собой компромисс между ними и предполагает менее строгую систему перераспределения, чем $B$, в то же время в меньшей степени тормозя рост всей экономики, чем В. Грэг согласен и с аргументами утилитаризма и с аргументами Ролза, но не может на этом основании выбрать режим А или В. Он выбирает $C$, хотя не разделяет этических оснований такого решения. Грэг выбирает $C$, потому что этот режим является вторым наилучшим и для утилитаристов, и для сторонников теории справедливости Ролза (Pittard, Worsnip, 2017: 169-170).

Как мы видим, в первом случае эквивалентом дескриптивной неопределенности служит незнание некоторых истинных утверждений (недостаток информации о некотором положении дел). Во-втором, нормативная неопределенность связана с тем, что действующий агент не может решить, что же является правильным, какие основания существуют у долженствования. Наиболее просто представить этот вид неопределенности как разделение мнений в рамках коллективного принятия решений, в условиях, когда все 
члены коллектива обладают одинаковой информацией, но разными нормативными установками. Каждый из них выбирает разные классы возможных миров, в которых происходит то, что считается должным. В случае дескриптивной неопределенности самопротиворечие (неконсистентность) может наступать в рассуждениях агента при переходе от дескриптивных суждений (о положении дел) к суждениям об оптимальном действии. Существует мнение, что из дизъюнкции «Или В - лучшее средство, или С - лучшее средство» в случае Ханны не следует суждение «Я должна дать пострадавшему или В, или С» (Kolodny, MacFarlane, 2010).

В рамках нормативной неопределенности противоречия наступают еще раньше по ходу рассуждения. Суждение «Я признаю верным или утилитаризм, или теорию справедливости Ролза» уже говорит о самопротиворечивости агента. Преодолеть такое противоречие и устранить неопределенность невозможно только за счет предоставления агенту дополнительной информации.

\section{ДЕСКРИПТИВНАЯ НЕОПРЕДЕЛЕННОСТЬ В МЕДИЦИНЕ: КОНЦЕПТУАЛИЗАЦИЯ И СТРАТЕГИИ ПРЕОДОЛЕНИЯ}

То, что выше описано как дескриптивная неопределенность, довольно давно и активно обсуждается как проблема в сфере организации здравоохранения (Babrow, Kasch, Ford, 1998). Не вникая в технические детали, обозначим основные ее источники - для прорисовки достаточно широкой перспективы практического аспекта проблемы неопределенности в медицине, а не для погружения в методологические и эпистемологические ее аспекты. Хан с соавторами выделяет три свойства информации в медицине, которые определяют заложенную в ней неопределенность: вероятностный характер информации, ее двусмысленность или сложность (Han, Klein, Arora, 2011). Но, как правило, в каждом случае эти факторы сочетаются в рамках одной ситуации, к тому же такая классификация прямо не соотносится с практиками минимизации неопределенности.

Исходя из необходимости такого практического соотнесения, обозначим, что источниками дескриптивной неопределенности могут быть: а) устранимое неведение, незнание каких-либо аспектов медицинской науки (например, вызванное пробелом в образовании — как в случае с Ханной); b) неведение, вызванное отсутствием достаточно точных способов узнать или распознать положение дел (например, наличием критически важных погрешностей средств диагностики); с) стохастический характер процесса, о будущем течении которого необходимо вынести суждение. Последнее - наиболее общий случай неопределенности, обсуждаемой в синергетике и теории сложности. Для медицины оценки вероятности таких процессов возможны исходя из статистических данных. Например, истинной может являться пропозиция «Медианная выживаемость больных раком молочной железы составляет 8 лет». Но истинной же может быть и пропозиция «Больше половины пациенток с тройным негативным подтипом рака молочной железы умирают в первые 3 года после постановки диагноза». Т. е. статистическая оцен- 
ка вероятности возможна, но она зависит от выбранных границ группы, к которой причисляют пациента, и для которой известно статистическое распределение интересующего параметра. Границы могут быть обозначены исходя из пола, возраста, истории болезни, а также - как показано в примере с раком молочной железы - исходя из классификации заболеваний.

Способы минимизации дескриптивной неопределенности в медицине могут быть сопоставлены с ее источниками. Неведение типа А - и, соответственно, дескриптивная невероятность, исходящая, например, от незнания Ханны - может быть преодолено за счет стандартизации медицинского образования, подготовке подробных клинических рекомендаций. Источник неопределенности типа В может быть устранен за счет валидации методов диагностики, калибровки ее инструментов, а также проведения повторных диагностических процедур. Неопределенность, связанную со сложностью и/или стохастическим характером исследуемых процессов Остин Баброу и Кимберли Клайн называют внутренне присущей или «врожденной» (inherent uncertainty) для процессов постановки диагноза и прогнозирования исходов лечения (Babrow, Kline, 2000). Соответственно, они предлагают врачу и пациенту оставить безуспешные попытки преодолеть ее и вместо этого научиться «справляться» или «ладить» с такой неопределенностью. Проще говоря - научиться жить с осознанием неопределенности, понимая ее как свойство, внутренне присущее самой жизни. Другие формы дескриптивной неопределенности они относят к «информационной неопределенности», вместе с тем выделяя три источника интерпретативной неопределенности. Последняя может быть связана с тем, что врач в точности не знает, во-первых, как пациент структурирует полученную от него информацию, во-вторых, ответы на какие вопросы пациент желает получить в рамках приема (или благодаря диагностической процедуре), в-третьих, неизвестно, насколько пациент готов столкнуться с дескриптивной неопределенностью. Однако представляется, что такое различение не обладает ни логической строгостью (семантические аспекты проблемы смешаны с прагматическими), ни практической релевантностью (выделены скорее отдельные тематические блоки в коммуникации врача и пациента, а не проблемы, связанные с коммуникативными практиками).

Подход к неопределенности в медицине, предложенный О. Баброу и К. Клайн интересен прежде всего их критикой «идеологии преодоления неопределенности» и вниманием к способам, позволяющим пациентам научиться жить с неопределенностью. В то же время предложенные ими этические регулятивы оставляют в стороне проблему нормативной неопределенности.

\section{ВЗАИМОНАЛОЖЕНИЕ НОРМАТИВНОЙ И ДЕСКРИПТИВНОЙ НЕОПРЕДЕЛЕННОСТИ}

Медико-генетическая помощь является сферой медицины, в которой нормативная неопределенность проявляется наиболее явно. В рамках ее оказания интересы пациента могут открыто противоречить интересам его кров- 
ных родственников. Согласно одному из четырех принципов биоэтики, признанных биомедицинских сообществом практически повсеместно, пациент обладает автономией, предполагающей среди прочего право распоряжаться всеми данными о своем здоровье. Однако согласно принципу «делай благо» врач обязан использовать всю имеющуюся у него информацию на благо людей, например, ради предотвращения развития болезней (Beauchamp, Childress, 2001). Немецкие специалисты по биоэтике Кристиан Ленк и Дебора Фроммельд предлагают рассмотреть довольно типичной для медицинской генетики казус, в рамках которого следование одному принципу исключает следование другому. 68-летняя пациентка с диагностированным наследственным неполипозным колоректальным раком отказывается информировать свою сестру и ее детей о возможных наследственных рисках из-за давнего семейного конфликта (Lenk, Frommeld, 2015). Существуют противоположные ответы на вопрос: «Должен ли врач вопреки запрету пациентки контактировать с ее родственниками?». Ответ «нет» предполагает ссылку на примат сохранения врачебной тайны и принцип автономии. Ответ «да» может быть этически обоснованным через признание семьи пациентки субъектом, которому оказывается медицинская помощь.

На первый взгляд, нормативная неопределенность преодолевается через установление законодательных норм и запретов. Однако, как пишут К. Ленк и Д. Фроммельд, если данный случай происходит в Германии, тогда врач не имеет права распространять информацию под угрозой тюремного заключения. В Великобритании у врача есть легальная возможность сделать это. Сложность заключается не в том, что нормативная неопределенность в разных юрисдикциях преодолевается по-разному. Скорее, проблема несколько глубже, и установление правовых регулятивов не всегда способно справиться с нормативной неопределенностью. Представим, что Ханна, героиня мысленного эксперимента, изложенного в начале статьи, вообще не имеет действующей врачебной лицензии. Придает ли это приоритет долженствованию «Вообще не оказывай помощь» над применением препарата С или препарата В? Заставляет ли это отбросить спасение человека с минимальным вредом его здоровью как стандарт оценки деяния Ханны? Нормативная неопределенность связана с тем, что сам агент не может отдать предпочтение одному из нескольких противоречащих стандартам оценки деяния, а не с действующими юридическими регулятивами.

В рамках геномной медицины могут возникнуть ситуации, в которых нормативная неопределенность вообще не может быть отодвинута на второй план правовыми регулятивами. Подобная ситуация возникает при решении вопроса об информировании пациента о результатах исследования, выявившего генетические варианты неопределенного значения (Ижевская, 2015). Описаны десятки вариантов для хорошо изученных генов, чья патогенетическая роль на момент обнаружения их в геноме пациентов была (а часто и остается) неизвестной.

С одной стороны, у врача есть основания предполагать, что эти особенности могут быть связаны с определенными рисками для здоровья паци- 
ента. С другой, эти риски не являются статистически валидированными, и их характер не до конца ясен. Неопределенное клиническое значение обнаруженных генетических последовательностей является источником дескриптивной неопределенности. Масштабное популяционное исследование могло бы определить границы степени риска, статистически связанного с конкретной генетической особенностью.

Но проблема выбора линии поведения в этом случае связана не только с дескриптивной неопределенностью. Врачу необходимо решить, является ли предпочтительным для пациента узнать о дескриптивной неопределенности (о потенциальном риске, который невозможно определить) или не узнавать о них, живя более спокойной жизнью. Фактически врач выбирает стандарт благополучия для пациента. Врач может отдать предпочтение автономии пациента, понятой как возможность извлечь благо из любой информации, или заботе о моральном и эмоциональном благополучии пациента. При этом обе альтернативы располагают серьезным этическим обоснованием в рамках концепций, которые нидерландский медицинский антрополог Эннмари Мол маркировала как «логика выбора» и «логика заботы» (Mol, 2008).

\section{«ЭТИКА НЕОПРЕДЕЛЕННОСТИ» В МЕДИЦИНСКОЙ ГЕНЕТИКЕ}

В приведенном выше примере нормативная неопределенность оказывается связана с выбором стратегии обращения с дескриптивной неопределенностью. Принимаемое в таких случаях регуляторное решение не снимает проблему неопределенности, устанавливая лишь границы дозволенных действий врача. Например, врач-генетик обязан сообщать пациенту о неожиданных находках только в 56 генах. В этом решении видна попытка перевода нормативной неопределенности в дескриптивную, так как такое разделение генов на «значимые» и «не значимые» для пациента лишь отражает уровень текущего медико-генетического знания, но не нормативный консенсус во врачебном сообществе. Попытку свести нормативную неопределенность к интерпретативной можно усмотреть в указании на «врачебное усмотрение» относительно блага пациента при решении вопроса об информировании пациента о вариантах неопределенного значения (Hawkins, Ho, 2012). Однако такое усмотрение может основываться только на осознаваемых (но не обязательно разделяемых) врачом ценностях, которые нуждаются в проговаривании. Одним из вариантов обсуждения ценностных оснований работы с дескриптивной неопределенностью является медицинская этика неопределенности. Благодаря артикуляции принципов и ценностей как опорных точек обсуждения она позволяет врачу не минимизировать, но «ладить» с нормативной неопределенностью. А на этом основании врач и пациент могут совместно «справиться», «сладить» с дескриптивной неопределенностью, присущей способам диагностики и прогнозируемым процессам.

Одна из возможных формулировок этики неопределенности предложена генетиками и специалистами по биоэтике из Сиднейского университета (Newson et al., 2016). Основными ее ориентирами служат устойчивость, бла- 
гополучие, автономия и солидарность (resilience, welfare, autonomy and solidarity). Устойчивость здесь предполагает понимание медико-генетической помощи не как однократного события консультирования, а как наличия постоянной возможности для пациента получить помощь в процессе осознания результатов, а для врача - информировать пациента о новых данных, устанавливающих значение обнаруженных у него генетических вариантов. Благополучие означает ориентацию на немедицинские характеристики состояния пациента, понимание, что сам по себе факт знакомства с генетическими рисками может нанести вред пациенту. Соответственно процесс коммуникации должен учитывать не только «биологический», но и «биографический» контекст жизни пациента. Автономия означает не возможность обречь пациента на самостоятельное переживание неопределенности, а поддержку со стороны врача критической рефлексии пациента о значении этой неопределенности. Наконец, солидарность предполагает разделение связанных с неопределенностью «издержек» между пациентом и его семьей, с одной стороны, и врачом и системой здравоохранения, с другой.

\section{СПИСОК ЛИТЕРАТУРЫ}

Ижевская, В. Л. (2015) Этические проблемы клинического применения генетического тестирования нового поколения // Рабочие тетради по биоэтике. Вып. 21: Философско-антропологические основания персонализированной медицины (междисциплинарный анализ) : сб. науч. ст. / под ред. П. Д. Тищенко. М. : Изд-во Моск. гуманит. ун-та. 208 с. С. 119-136.

Babrow, A. S., Kasch, C. R., Ford, L. A. (1998) The many meanings of uncertainty in illness: toward a systematic accounting // Health Communication. Vol. 10. Issue 1. P. 1-23. DOI: $10.1207 / \mathrm{s} 15327027 h c 1001 \_1$

Babrow, A. S., Kline, K. N. (2000) From "reducing” to "coping with" uncertainty: Reconceptualizing the central challenge in breast self-exams // Social Science and Medicine. Vol. 51. No. 12. P. 1805-1816.

Beauchamp, T. L., Childress, J. F. (2001) Principles of biomedical ethics. 5th ed. Oxford : Oxford University Press. xi, 454 p.

Dowell, J. (2012) Contextualist solutions to three puzzles about practical conditionals // Oxford Studies in Metaethics. Vol. 7 / ed. by R. Shafer-Landau. Oxford : Clarendon Press. 384 p. P. 271-303. DOI: 10.1093/acprof:oso/9780199653492.

$\underline{003.0009}$

Han, P. K., Klein, W. M., Arora, N. K. (2011) Varieties of uncertainty in health care: A conceptual taxonomy // Medical Decision Making. Vol. 31. No. 6. P. 828-838.

Hawkins, A. K., Ho, A. (2012) Genetic counseling and the ethical issues around direct to consumer genetic testing // Journal of Genetic Counseling. Vol. 21. No. 3. P. 367-373. DOI: $10.1007 /$ s10897-012-9488-8

Jackson, F. (1991) Decision-theoretic consequentialism and the nearest and dearest objection // Ethics. Vol. 101. No. 3. P. 461-482. DOI: $10.1086 / 293312$ 
Kolodny, N., MacFarlane, J. (2010) Ifs and oughts // The Journal of Philosophy. Vol. 107. No. 3. P. 115-143. DOI: 10.5840/jphil2010107310

Lenk, C., Frommeld, D. (2015) Different concepts and models of information for family-relevant genetic findings: comparison and ethical analysis // Medicine, Health Care and Philosophy. Vol. 18. Issue 3. P. 393-408. DOI: 10.1007/s11019-015-9638-5

Mol, A. (2008) The logic of care: Health and the problem of patient choice. L. ; N. Y. : Routledge Press. xii, 129 p.

Newson, A. J. et al. (2016) Known unknowns: building an ethics of uncertainty into genomic medicine / A. J. Newson, S. J. Leonard, A. Hall, C. L. Gaff // BMC Medical Genomics. Vol. 9. No. 1. Article 57. DOI: 10.1186/s12920-0160219-0

Pittard, J., Worsnip, A. (2017) Metanormative contextualism and normative Uncertainty // Mind. Vol. 126. No. 501. P. 155-193. DOI: 10.1093/mind/fzv208

Дата поступления: 21.10.2018 2.

\section{REFERENCES}

Izhevskaya, V. L. (2015) Eticheskie problemy klinicheskogo primeneniia geneticheskogo testirovaniia novogo pokoleniia [Ethical challenges of clinical application of next generation sequencing]. In: Rabochie tetradi po bioetike [Workbooks on bioethics]. Vol. 21: Filosofsko-antropologicheskie osnovaniia personalizirovannoi meditsiny (mezhdistsiplinarnyi analiz) [Philosophical and anthropological foundations of personalized medicine (interdisciplinary analysis)] : A collection of articles / ed. by P. D. Tishchenko. Moscow : Moscow University for the Humanities Publ. 208 p. Pp. 119-136. (In Russ.).

Babrow, A. S., Kasch, C. R. and Ford, L. A. (1998) The many meanings of uncertainty in illness: toward a systematic accounting. Health Communication, vol. 10, issue 1, pp. 1-23. DOI: 10.1207/s15327027hc1001_1

Babrow, A. S. and Kline, K. N. (2000) From "reducing” to “coping with" uncertainty: Reconceptualizing the central challenge in breast self-exams. Social Science and Medicine, vol. 51, no. 12, pp. 1805-1816.

Beauchamp, T. L. and Childress, J. F. (2001) Principles of biomedical ethics. 5th ed. Oxford : Oxford University Press. xi, 454 p.

Dowell, J. (2012) Contextualist solutions to three puzzles about practical conditionals. In: Oxford Studies in Metaethics. Vol. 7 / ed. by R. Shafer-Landau. Oxford : Clarendon Press. 384 p. Pp. 271-303. DOI: 10.1093/acprof:oso/97 80199653492.003.0009

Han, P. K., Klein, W. M. and Arora, N. K. (2011) Varieties of uncertainty in health care: A conceptual taxonomy. Medical Decision Making, vol. 31, no. 6, pp. 828-838.

Hawkins, A. K. and Ho, A. (2012) Genetic counseling and the ethical issues around direct to consumer genetic testing. Journal of Genetic Counseling, vol. 21, no. 3, pp. 367-373. DOI: 10.1007/s10897-012-9488-8 
Jackson, F. (1991) Decision-theoretic consequentialism and the nearest and dearest objection. Ethics, vol. 101, no. 3, pp. 461-482. DOI: 10.1086/293312

Kolodny, N. and MacFarlane, J. (2010) Ifs and oughts. The Journal of Philosophy, vol. 107, no. 3, pp. 115-143. DOI: 10.5840/jphil2010107310

Lenk, C. and Frommeld, D. (2015) Different concepts and models of information for family-relevant genetic findings: comparison and ethical analysis. Medicine, Health Care and Philosophy, vol. 18, issue 3, pp. 393-408. DOI: 10.1007/s11019-015-9638-5

Mol, A. (2008) The logic of care: Health and the problem of patient choice. London ; New York : Routledge Press. xii, 129 p.

Newson, A. J. et al. (2016) Known unknowns: building an ethics of uncertainty into genomic medicine / A. J. Newson, S. J. Leonard, A. Hall and C. L. Gaff. BMC Medical Genomic, vol. 9, no. 1, article 57. DOI: 10.1186/s12920-016-0219-0

Pittard, J. and Worsnip, A. (2017) Metanormative contextualism and normative Uncertainty. Mind, vol. 126, no. 501, pp. 155-193. DOI: 10.1093/mind/fzv208

Submission date: 21.10.2018.

Шевченко Сергей Юрьевич - младший научный сотрудник сектора гуманитарных экспертиз и биоэтики Института философии Российской академии наук. Адрес: 109240, г. Москва, ул. Гончарная, д. 12, стр. 1. Тел.: +7 (495) 697-90-67. Эл. адрес: simurg87@list.ru

Шестак Анна Геннадьевна - научный сотрудник Российского научного центра хирургии имени академика Б. В. Петровского. Адрес: 119991, г. Москва, ГСП-1, Абрикосовский пер., д. 2. Эл. адрес: anna.shestak87@gmail. com

Shevchenko Sergei Yurievich, Junior Research Fellow, Department of Humanitarian Expertise and Bioethics, Institute of Philosophy, Russian Academy of Sciences. Postal address: Bldg. 1, 12 Goncharnaya St., 109240 Moscow, Russian Federation. Tel.: +7 (495) 697-90-67. E-mail: simurg87@list.ru

ORCID: 0000-0002-7935-3444

Researcher ID: F-5320-2018

Scopus Author ID: 57192993265

SPIN-код: 2783-6006

Shestak Anna Gennadievna, Researcher, Petrovsky National Research Centre of Surgery. Postal address: 2 Abrikosovsky Lane, 119991 Moscow, Russian Federation. E-mail: anna.shestak87@gmail.com

\section{Для ичитирования:}

Шевченко С. Ю., Шестак А. Г. Нормативная и дескриптивная неопределенность в геномной медицине [Электронный ресурс] // Горизонты гуманитарного знания. 2019. № 1. C. 120-130. URL: http://journals.mosgu.ru/ ggz/article/view/948 (дата обращения: дд.мм.гггг). DOI: 10.17805/ggz.2019.1.7 